\title{
Structure of the vacuum in deformed supersymmetric chiral models
}

\author{
P. Fernández, ${ }^{a}$ E.F. Moreno ${ }^{b}$ and F.A. Schaposnik ${ }^{a *}$ \\ a Departamento de Física, Universidad Nacional de La Plata, \\ C.C. 67, 1900 La Plata, Argentina \\ ${ }^{b}$ Department of Physics, West Virginia University, \\ Morgantown, West Virginia 26506-6315, U.S.A. \\ E-mail: pfernandez@fisica.unlp.edu.ar, Enrique.Moreno@mail.wvu.edu, \\ fidel@fisica.unlp.edu.ar
}

ABSTRACT: We analyze the vacuum structure of $\mathcal{N}=1 / 2$ chiral supersymmetric theories in deformed superspace. In particular we study O'Raifeartaigh models with $C$-deformed superpotentials and canonical and non-canonical deformed Kähler potentials. We find conditions under which the vacuum configurations are affected by the deformations.

KEYWords: Supersymmetry Breaking, Non-Commutative Geometry.

${ }^{*}$ Associated with CICBA 


\section{Contents}

1. Introduction 1

2. Non(anti)commutative superspace and chiral models 2

2.1 The setting 2

2.2 Chiral models 3

2.3 Vacuum properties in deformed theories

3. Deformed O'Raifeartaigh models 5

3.1 Two specific cases

3.2 A more general superpotential 9

4. Noncanonical deformed Kähler potentials 10

5. Discussion 12

\section{Introduction}

A $C$-deformation of the $\mathcal{N}=1$ superalgebra corresponding to nonanticommutative Grassmann coordinates $\theta^{\alpha}$ has been shown to arise in string theory in a graviphoton background [1] $]$ [2]. Prompted by this result, nonanticommutative versions of supersymmetric (SUSY) Yang-Mills theory and Wess-Zumino model have been formulated [3]-四] and their renormalizability established [5]-[7]. The deformation preserves the notion of chirality but only half of the $\mathcal{N}=1$ supersymmetry is preserved as the supercharges $Q_{\alpha}$, the generators of $\theta_{\alpha}$ translations, are conserved while the $\bar{Q}_{\dot{\alpha}}$ are broken explicitly.

In order to analyze the vacuum structure of undeformed SUSY chiral models we study the effective potential $V$ for scalar fields since its critical points correspond to the possible vacua. Hermiticity of the original theory guarantees that the resulting potential is positive definite so that the vanishing of $V$ implies the existence of a supersymmetric vacuum. But in $C$-deformed SUSY theories hermiticity is lost, $V$ is not positive definite and the analysis of the critical points should be done at the quantum level using saddle point or steepest descent methods.

The issue of spontaneous supersymmetry breaking in O'Raifeartaigh models [8] has recently received much attention after the discovery of meta-stable SUSY breaking vacua in $\mathcal{N}=1$ SQCD that can be seen, in the low-energy effective theory, as vacua of an O'Raifeartaigh-type model [9- 14]. In connection with this phenomenon, it is the purpose of this work to analyze the structure of the vacuum for $C$-deformed O'Raifeartaigh-like models, discussing in particular the possibility of spontaneous breaking of the surviving supersymmetry. 
As explained in [6] a $\mathcal{N}=1 / 2$ supersymmetric vacuum requires both the $\mid$ vac $\rangle$ state and its dual $\langle\mathrm{vac}|$ to be annihilated by $Q_{\alpha}$. This is connected to the fact that the vacuum energy of such state, $\langle\mathrm{vac}|E| \mathrm{vac}\rangle$, vanishes even if the energy associated with the non-Hermitian deformed Lagrangian is in general complex-valued. Hence, the analysis of the zeroes of the scalar potential still provides information about symmetry breaking in deformed models and this is the route we will follow in this investigation.

A discussion of the scalar potential for certain SUSY deformed models has been already presented in refs. [15] 18 for deformed Wess-Zumino and sigma models (with canonical Kähler potentials). Here we will consider O'Raifeartaigh models with more general deformed superpotentials and we will also discuss the case of deformed non-canonical Kähler potentials. The plan of the paper is the following: In section 2 we establish our conventions for nonanticommutative superspace and present general deformed models containing chiral superfields. In section 3 we analyze the vacuum structure of rather general deformed O'Raifeartaigh-like models in which the Kähler potential is kept canonical, and in section 4 a similar analysis of deformed models with non-canonical Kähler potential. We summarize and discuss our results in section 5 .

\section{Non(anti)commutative superspace and chiral models}

\subsection{The setting}

We consider the deformation of 4 dimensional Euclidean $\mathcal{N}=1$ superspace parametrized by superspace bosonic coordinates $x^{\mu}$ and chiral and anti-chiral fermionic coordinates $\theta^{\alpha}, \bar{\theta}^{\dot{\alpha}}$ as proposed in [3]

$$
\begin{array}{ll}
\left\{\theta^{\alpha}, \theta^{\beta}\right\}=C^{\alpha \beta} & \\
\left\{\bar{\theta}^{\dot{\alpha}}, \bar{\theta}^{\dot{\beta}}\right\}=0, & \left\{\theta^{\alpha}, \bar{\theta}^{\dot{\beta}}\right\}=0
\end{array}
$$

Here $C^{\alpha \beta}$ are constant elements of a symmetric matrix. Defining chiral and anti-chiral coordinates according to

$$
\begin{aligned}
& y^{\mu}=x^{\mu}+i \theta \sigma^{\mu} \bar{\theta} \\
& \bar{y}^{\mu}=y^{\mu}-2 i \theta \sigma^{\mu} \bar{\theta}
\end{aligned}
$$

we impose

$$
\left[y^{\mu}, y^{\nu}\right]=\left[y^{\mu}, \theta^{\alpha}\right]=\left[y^{\mu}, \bar{\theta}^{\dot{\alpha}}\right]=0
$$

and obtain, as a consequence of (2.1)-(2.5),

$$
\left[\bar{y}^{\mu}, \bar{y}^{\nu}\right]=4 \bar{\theta} \bar{\theta} \mathbf{C}^{\mu \nu}
$$

where $\mathbf{C}^{\mu \nu}=C^{\alpha \beta}\left(\sigma^{\mu \nu}\right)_{\alpha \beta}$ is antisymmetric and antiselfdual.

The non(anti)commutative field theory in such a deformed superspace can be defined in terms of superfields that are multiplied according to the following Moyal product [3]

$$
\Phi(y, \theta, \bar{\theta}) * \Psi(y, \theta, \bar{\theta})=\Phi(y, \theta, \bar{\theta}) \exp \left(-\frac{C^{\alpha \beta}}{2} \frac{\overleftarrow{\partial}}{\partial \theta^{\alpha}} \frac{\vec{\partial}}{\partial \theta^{\beta}}\right) \Psi(y, \theta, \bar{\theta})
$$


Supercharges and covariant derivatives in chiral coordinates take the form

$$
\begin{array}{ll}
Q_{\alpha}=\frac{\partial}{\partial \theta^{\alpha}}, & \bar{Q}_{\dot{\alpha}}=-\frac{\partial}{\partial \bar{\theta}^{\dot{\alpha}}}+2 i \theta^{\alpha} \sigma_{\alpha \dot{\alpha}}^{\mu} \frac{\partial}{\partial y^{\mu}}, \\
D_{\alpha}=\frac{\partial}{\partial \theta^{\alpha}}+2 i \sigma_{\alpha \dot{\alpha}}^{\mu} \bar{\theta}^{\dot{\alpha}} \frac{\partial}{\partial y^{\mu}}, & \bar{D}_{\dot{\alpha}}=-\frac{\partial}{\partial \bar{\theta}^{\dot{\alpha}}}
\end{array}
$$

The $D-D$ algebra is not modified by the deformation (2.1) as it also happens for the $Q-D$ and $\bar{Q}-D$ algebra. Concerning the supercharge algebra, it is modified according to

$$
\begin{aligned}
& \left\{\bar{Q}_{\dot{\alpha}}, Q_{\alpha}\right\}=2 i \sigma_{\alpha \dot{\alpha}}^{\mu} \frac{\partial}{\partial y^{\mu}}=2 \sigma_{\alpha \dot{\alpha}}^{\mu} P_{\mu} \\
& \left\{Q_{\alpha}, Q_{\beta}\right\}=0 \\
& \left\{\bar{Q}_{\dot{\alpha}}, \bar{Q}_{\dot{\beta}}\right\}=-4 C^{\alpha \beta} \sigma_{\alpha \dot{\alpha}}^{\mu} \sigma_{\beta \dot{\beta}}^{\nu} \frac{\partial^{2}}{\partial y^{\mu} \partial y^{\nu}}=4 C^{\alpha \beta} \sigma_{\alpha \dot{\alpha}}^{\mu} \sigma_{\beta \dot{\beta}}^{\nu} P_{\mu} P_{\nu}
\end{aligned}
$$

Then, only the subalgebra generated by $Q_{\alpha}$ is still preserved and this defines the chiral $\mathcal{N}=1 / 2$ supersymmetry algebra 3 .

\subsection{Chiral models}

In this work we will discuss models containing chiral superfields. In deformed superspace, a chiral superfield $\Phi$ satisfying $\bar{D}_{\dot{\alpha}} \Phi=0$ can be written, as usual, in the form

$$
\Phi(y, \theta)=\phi(y)+\sqrt{2} \theta \psi(y)+\theta \theta F(y)
$$

Analogously we can define antichiral superfields satisfying

$$
D_{\alpha} \bar{\Phi}=0
$$

which only depend on $\bar{\theta}$ and $\bar{y}^{\mu}$.

A general action in terms of chiral and antichiral superfields takes the form

$$
S[\Phi, \bar{\Phi}]=\int d^{4} y\left[\int d^{2} \theta d^{2} \bar{\theta} K_{*}\left(\Phi^{i}, \bar{\Phi}^{\bar{j}}\right)+\int d^{2} \theta W_{*}\left(\Phi^{i}\right)+\int d^{2} \bar{\theta} \bar{W}_{*}\left(\bar{\Phi}^{\bar{j}}\right)\right]
$$

Here we call $K_{*}, W_{*}$ the Kähler and superpotential functionals with superfields multiplied using the Moyal product. A very useful formula for handling these quantities has been derived in [16] [17]. For example, given the superpotential $W_{*}(\Phi)$, we can define a "diffuse superpotential"

$$
\widetilde{\mathcal{W}}\left(\phi_{i}, F_{i}\right)=\int_{-1}^{1} d \xi \mathcal{W}\left(\phi_{i}+\xi c F_{i}\right)
$$

where fields $\phi_{i}$ are multiplied in the r.h.s. with the ordinary product and we have written $c=\sqrt{-\operatorname{det} C}$. As pointed out in [16], non(anti)commutativity induces certain fuzziness controlled by auxiliary fields $F_{i}$.

Using eq. (2.16), we can prove that, in terms of component fields, the scalar potential can be written

$$
V_{\text {scalar }}\left(\phi_{i}, \bar{\phi}_{\bar{i}}\right)=\frac{1}{2} F_{i} \widetilde{W},\left.{ }_{i}\right|_{F_{i}=F_{i}(\phi, \bar{\phi})}
$$


with all products being ordinary products. Analogously, we can define, starting from the Kähler potential, the following diffuse quantities [17]

$$
\begin{aligned}
Z(\phi, \bar{\phi}, F)= & \int_{-1}^{1} d \xi K\left(\phi_{i}+\xi c F_{i}, \bar{\phi}_{\bar{j}}\right) \\
Y(\phi, \bar{\phi}, F, \bar{F})= & \bar{F}_{\bar{p}} Z{ }_{, \bar{p}}-\frac{1}{2}\left(\bar{\chi}_{\bar{p}} \bar{\chi}_{\bar{q}} Z{ }_{\bar{p} \bar{q}}\right) \\
& +c \int_{-1}^{1} d \xi \xi\left[\partial^{\mu} \bar{\phi}_{\bar{p}} \partial_{\mu} \bar{\phi}_{\bar{q}} K{ }_{\bar{p} \bar{q}}^{\xi}+\nabla^{2} \bar{\phi}_{\bar{p}} K_{\bar{p}}^{\xi}\right]
\end{aligned}
$$

Now, calling

$$
\int d^{4} y L_{K} \equiv \int d^{4} y \int d^{2} \theta d^{2} \bar{\theta} K_{*}\left(\Phi^{i}, \bar{\Phi}^{\bar{j}}\right)
$$

it can be shown that

$$
\begin{aligned}
L_{K}= & \frac{1}{2} F_{i} Y,_{i}+\frac{1}{2} \partial^{\mu} \bar{\phi}_{\bar{p}} \partial_{\mu} \bar{\phi}_{\bar{q}} Z_{, \bar{p} \bar{q}}+\frac{1}{2} \nabla^{2} \bar{\phi}_{\bar{p}} Z_{, \bar{p}}-\frac{1}{4}\left(\chi^{i} \chi^{j}\right) Y_{, i j} \\
& -\frac{1}{2} i\left(\chi^{i} \sigma^{\mu} \bar{\chi}^{\bar{p}}\right) \partial_{\mu} \bar{\phi}_{\bar{q}} Z_{, i \bar{p} \bar{q}}-\frac{1}{2} i\left(\chi^{i} \sigma^{\mu} \partial_{\mu} \bar{\chi}^{\bar{p}}\right) Z_{, i \bar{p}}
\end{aligned}
$$

\subsection{Vacuum properties in deformed theories}

The choice of deforming the anticommutator of $\theta_{\alpha}$ (2.1), without altering that of $\bar{\theta}_{\dot{\alpha}}$ implies that $\bar{\theta}_{\dot{\alpha}}$ are not the complex conjugate of $\theta_{\alpha}$, which is only possible in Euclidean space. Moreover, hermiticity of the theory is lost because of the deformation and then the usual analysis of the the potential minima should be replaced by a careful analysis of the critical points of the resulting complex expression. At the quantum level, saddle point or steepest descent methods should be applied as usual, but taking into account that trajectories are in principle complex and that space is Euclidean.

As shown in ref. [6] taking the deformed Wess-Zumino model as a prototype of $\mathcal{N}=1 / 2$ theories with chiral superfields, the vacuum energy, computed from the effective action for constant bosonic fields, vanishes

$$
\langle\operatorname{vac}|E| \operatorname{vac}\rangle=\left\langle\operatorname{vac}\left|Q_{\alpha} \bar{Q}_{\dot{\alpha}}+\bar{Q}_{\dot{\alpha}} Q_{\alpha}\right| \operatorname{vac}\right\rangle=0
$$

Then, in order to have a supersymmetric vacuum $Q_{\alpha}$, the generator of the surviving supersymmetry, should annihilate both $|\mathrm{vac}\rangle$ and $\langle\mathrm{vac}|$,

$$
Q_{\alpha}|\operatorname{vac}\rangle=0, \quad\langle\operatorname{vac}| Q_{\alpha}=0
$$

since, being $\bar{Q}_{\dot{\alpha}}$ the generator of the explicitly broken supersymmetry, $\bar{Q}_{\dot{\alpha}} \mid$ vac $\rangle$ does not vanish in general.

Vanishing of the vacuum energy for supersymmetric vacua is not a consequence of any specific choice of the deformed superpotential. As explained in ([6]), supersymmetric vacua in deformed models with chiral fields impose the condition $\partial \bar{W}_{*}(\bar{\Phi}) / \partial \bar{\Phi}=0$ which in turn imply the vanishing of the corresponding scalar potential. 


\section{Deformed O'Raifeartaigh models}

We discuss here how the landscape of extrema of the scalar potential in O'Raifeartaigh models is affected by the deformation of superspace defined in eq. (2.1).

\subsection{Two specific cases}

Consider three chiral superfields fields $\Phi_{i}(i=1,2,3)$ and a canonical Kähler potential $K=\bar{\Phi}_{i} * \Phi_{i}$. Concerning the superpotential, we choose

$$
\mathcal{W}=\Phi_{1} *\left(\frac{h}{2} \Phi_{3} * \Phi_{3}+f\right)+m \Phi_{2} * \Phi_{3}+\mathrm{ST}
$$

which has the typical O'Raifeartaigh potential form, extended to non(anti)commutative space. Here ST includes all necessary symmetrizing terms so that the potential is symmetrized with respect to the $*$ product. For simplicity, we take all parameters $(f, m, \ldots)$ as real numbers. In order to compute the scalar potential for component fields $\phi$ we use eq. (2.17). In view of the form of the superpotential, $\mathcal{W}\left(\phi_{i}+\xi c F_{i}\right)$ as defined in (2.16) will only have terms with powers $\xi^{n}, n=0,1,2,3$. Moreover, since integrals with odd powers in $\xi$ vanish we end with

$$
\mathcal{W}\left(\phi_{i}+\xi c F_{i}\right)=\phi_{1}\left(\frac{h}{2}\left(\phi_{3}\right)^{2}+\xi^{2} c^{2}\left(F_{3}\right)^{2}+f\right)+\xi^{2} c^{2} h \phi_{3} F_{3} F_{1}
$$

so the diffuse superpotential $\widetilde{\mathcal{W}}$ becomes

$$
\widetilde{\mathcal{W}}\left(\phi_{i}, F_{i}\right)=2 \phi_{1}\left(\frac{h}{2}\left(\phi_{3}\right)^{2}+f\right)+\frac{2}{3} c^{2}\left[\left(F_{3}\right)^{2}+h \phi_{3} F_{3} F_{1}\right]
$$

leading to a scalar potential

$$
V_{E}=F_{1}\left(\frac{h}{2}\left(\phi_{3}\right)^{2}+f\right)+m\left(F_{2} \phi_{3}+F_{3} \phi_{2}\right)+h F_{3} \phi_{1} \phi_{3}-\frac{\operatorname{det} C}{2} h F_{1}\left(F_{3}\right)^{2}
$$

The subscript $E$ indicates that we are dealing with the Euclidean potential which is minus the Minkowski potential.

Using the equations of motion to replace auxiliary fields $F_{i}$ and putting all fermion fields to zero we end with

$$
\begin{aligned}
V_{E}= & -\left(\frac{h}{2}\left(\phi_{3}\right)^{2}+f\right)\left(\frac{h}{2}\left(\bar{\phi}_{\overline{3}}\right)^{2}+f\right)-\left(h \phi_{1} \phi_{3}+m \phi_{2}\right)\left(h \bar{\phi}_{\overline{1}} \bar{\phi}_{\overline{3}}+m \bar{\phi}_{\overline{2}}\right) \\
& -m^{2} \phi_{3} \bar{\phi}^{\overline{3}}+\frac{h}{2} \operatorname{det} C\left(\frac{h}{2}\left(\bar{\phi}_{\overline{3}}\right)^{2}+f\right)\left(h \bar{\phi}_{\overline{1}} \bar{\phi}_{\overline{3}}+m \bar{\phi}_{\overline{2}}\right)^{2}
\end{aligned}
$$

For $C=0$ we recover the ordinary superspace result with a real potential provided $\phi^{*}=\bar{\phi}$. For $\operatorname{det} C \neq 0$ the potential becomes complex not only because the term proportional to $\operatorname{det} C$ is not accompanied by its complex conjugate but also because in principle $\bar{\phi}$ is not the complex conjugate of $\phi$. 
The equations for the extrema of potential (3.3) read

$$
\begin{aligned}
0= & h \phi_{3}\left(h \bar{\phi}_{\overline{1}} \bar{\phi}_{\overline{3}}+m \bar{\phi}_{\overline{2}}\right) \\
0= & m\left(h \bar{\phi}_{\overline{1}} \bar{\phi}_{\overline{3}}+m \bar{\phi}_{\overline{2}}\right) \\
0= & h \phi_{3}\left(\frac{h}{2}\left(\bar{\phi}_{\overline{3}}\right)^{2}+f\right)+m^{2} \bar{\phi}_{\overline{3}}+h \phi_{1}\left(h \bar{\phi}_{\overline{1}} \bar{\phi}_{\overline{3}}+m \bar{\phi}_{\overline{2}}\right) \\
0= & \left(h \phi_{1} \phi_{3}+m \phi_{2}\right) h \bar{\phi}_{\overline{3}} \\
& -\operatorname{det} C h\left(\frac{h}{2}\left(\bar{\phi}_{\overline{3}}\right)^{2}+f\right)\left(h \bar{\phi}_{\overline{1}} \bar{\phi}_{\overline{3}}+m \bar{\phi}_{\overline{2}}\right) h \bar{\phi}_{\overline{3}} \\
0= & m\left(h \phi_{1} \phi_{3}+m \phi_{2}\right)-\operatorname{det} C h m\left(\frac{h}{2}\left(\bar{\phi}_{\overline{3}}\right)^{2}+f\right)\left(h \bar{\phi}_{\overline{1}} \bar{\phi}_{\overline{3}}+m \bar{\phi}_{\overline{2}}\right) \\
0= & \left(\frac{h}{2}\left(\phi_{3}\right)^{2}+f\right) h \bar{\phi}_{\overline{3}}+\left(h \phi_{1} \phi_{3}+m \phi_{2}\right) h \bar{\phi}_{\overline{1}}+m^{2} \phi_{3} \\
& -\operatorname{det} C \frac{h}{2}\left(h \bar{\phi}_{\overline{3}}\left(h \bar{\phi}_{\overline{1}} \bar{\phi}_{\overline{3}}+m \bar{\phi}_{\overline{2}}\right)^{2}+2\left(\frac{h}{2}\left(\bar{\phi}_{\overline{3}}\right)^{2}+f\right)\left(h \bar{\phi}_{\overline{1}} \bar{\phi}_{\overline{3}}+m \bar{\phi}_{\overline{2}}\right) h \bar{\phi}_{\overline{1}}\right)
\end{aligned}
$$

Let us first consider the case $m \neq 0$. In this case, eq. (3.5) implies

$$
h \bar{\phi}_{\overline{1}} \bar{\phi}_{\overline{3}}+m \bar{\phi}_{\overline{2}}=0
$$

The l.h.s of this equation appears as a factor in all terms containing $\operatorname{det} C$ and hence all dependence on $C_{\alpha \beta}$ disappears. Field configurations corresponding to extrema of the potential are not affected by the deformation. Moreover, the value of the potential at the extrema is also unaffected by non(anti)commutativity since terms containing $\operatorname{det} C$ are multiplied by the same vanishing factor. The only difference with an ordinary superspace theory is that, in principle, $\bar{\phi}_{\bar{i}}$ does not necessarily coincide with $\phi_{i}^{*}$. For the particular field configurations where $\bar{\phi}_{\bar{i}}=\phi_{i}^{*}$, the results for the undeformed case [10] apply, and we can conclude that there is symmetry breaking, no runaway directions, and a classical pseudomoduli space with degenerate non supersymmetric vacua (arbitrary $\phi_{1}^{\text {vac }}$ ).

Concerning the general case in which $\bar{\phi}_{\bar{i}} \neq \phi_{i}^{*}$, we find extrema with similar properties as those with $\bar{\phi}_{\bar{i}}=\phi_{i}^{*}$ discussed above except that the pseudomoduli is spanned here by $\phi_{1}$ and $\bar{\phi}_{1}$ and hence its dimension is doubled. We conclude the discussion of the $m \neq 0$ case noting that the theory above corresponds to a generic supersymmetry breaking potential because the equation $V=0$ cannot be generically solved.

We will show that the situation changes when the coefficient $m$ in (3.1) vanishes. In that case the $\phi_{2}$ field decouples and the scalar potential takes the form

$$
\begin{aligned}
V_{E}= & -\left(\frac{h}{2}\left(\phi_{3}\right)^{2}+f\right)\left(\frac{h}{2}\left(\bar{\phi}_{\overline{3}}\right)^{2}+f\right)-h^{2} \phi_{1} \phi_{3} \bar{\phi}_{\overline{1}} \bar{\phi}_{\overline{3}} \\
& +\frac{h^{3}}{2} \operatorname{det} C\left(\frac{h}{2}\left(\bar{\phi}_{\overline{3}}\right)^{2}+f\right)\left(\bar{\phi}_{\overline{1}} \bar{\phi}_{\overline{3}}\right)^{2}
\end{aligned}
$$

In the undeformed case we can easily see that there exist two supersymmetric vacua which correspond to $\phi_{1}^{\mathrm{vac}}=0$ and $\phi_{3}= \pm \sqrt{-2 f / h}$ and a supersymmetry breaking flat direction for $\phi_{3}^{\mathrm{vac}}=0, \phi_{1}^{\mathrm{vac}}$ arbitrary, for which $V=f^{2}$ (in Minkowski space). 
In the deformed model there are also six families of supersymmetric configurations which do not depend on $\operatorname{det} C$. Namely

$$
\begin{array}{ll}
\bar{\phi}_{\overline{1}}=0, & \bar{\phi}_{\overline{3}}= \pm \sqrt{-\frac{2 f}{h}}, \\
\phi_{3}=0, & \bar{\phi}_{\overline{3}}= \pm \sqrt{-\frac{2 f}{h}} \\
\bar{\phi}_{\overline{3}}=0, & \phi_{3}= \pm \sqrt{-\frac{2 f}{h}}
\end{array}
$$

All other fields not included in each line are arbitrary.

Concerning non-supersymmetric extrema, they are the same for the undeformed and the deformed case,

$$
\phi_{3}=\bar{\phi}_{\overline{3}}=0, \quad \phi_{1} \text { and } \bar{\phi}_{\overline{1}} \text { arbitrary }
$$

and for these configurations $V_{E}=-f^{2}$.

There are also four solutions for which the fields at the extrema $\operatorname{depend}$ on $\operatorname{det} C$

$$
\phi_{1}=\phi_{3}=0, \quad \bar{\phi}_{\overline{1}}= \pm \frac{1}{h \sqrt{-\operatorname{det} C}}, \quad \bar{\phi}_{\overline{3}}= \pm \sqrt{-\frac{2 f}{h}}
$$

For these configurations $V=0$ and hence they correspond to supersymmetric vacua. A remarkable feature of these extrema can be seen by taking $\operatorname{det} C \in \mathbb{R}$. Indeed, in that case, in the $\operatorname{det} C \rightarrow 0^{+}$limit, they correspond to runaway directions which do not satisfy the extrema conditions of the undeformed potential. Hence, they have emerged entirely as a consequence of the deformation.

Let us now consider the vacua structure of another potential which results from the following superpotential

$$
\mathcal{W}=h \Phi_{1} * \Phi_{3} *\left(\Phi_{3}-m_{1}\right)+m \Phi_{2} *\left(\Phi_{3}-m_{1}\right)+\mathrm{ST}
$$

In contrast with the superpotential (3.1), the form of this superpotential allows for the existence of critical points $\partial \mathcal{W} / \partial \phi_{1}=\partial \mathcal{W} / \partial \phi_{2}=0$.

A completely analogous calculation to that presented above leads to the following expression for the scalar potential

$$
\begin{aligned}
V_{E}= & -\left(h \phi_{3}\left(\phi_{3}-m_{1}\right)\right)\left(h \bar{\phi}_{\overline{3}}\left(\bar{\phi}_{\overline{3}}-m_{1}\right)\right)-m\left(\phi_{3}-m_{1}\right) m\left(\bar{\phi}_{\overline{3}}-m_{1}\right) \\
& -\left[h \phi_{1}\left(2 \phi_{3}-m_{1}\right)+m \phi_{2}\right]\left[h \bar{\phi}_{\overline{1}}\left(2 \bar{\phi}_{\overline{3}}-m_{1}\right)+m \bar{\phi}_{\overline{2}}\right] \\
& +\operatorname{det} C h\left[h \bar{\phi}_{\overline{1}}\left(2 \bar{\phi}_{\overline{3}}-m_{1}\right)+m \bar{\phi}_{\overline{2}}\right]^{2}\left(h \bar{\phi}_{\overline{3}}\left(\bar{\phi}_{\overline{3}}-m_{1}\right)\right)
\end{aligned}
$$

The equations for the extrema of potential (3.18) read

$$
\begin{aligned}
0 & =\frac{\partial V}{\partial \phi_{1}}=h\left(2 \phi_{3}-m_{1}\right)\left[h \bar{\phi}_{\overline{1}}\left(2 \bar{\phi}_{\overline{3}}+m_{1}\right)+m \bar{\phi}_{\overline{2}}\right] \\
0 & =\frac{\partial V}{\partial \phi_{2}}=m\left[h \bar{\phi}_{\overline{1}}\left(2 \bar{\phi}_{\overline{3}}-m_{1}\right)+m \bar{\phi}_{\overline{2}}\right]
\end{aligned}
$$




$$
\begin{aligned}
0=\frac{\partial V}{\partial \phi_{3}}= & \left(2 h \phi_{3}-h m_{1}\right)\left(h \bar{\phi}_{\overline{3}}\left(\bar{\phi}_{\overline{3}}-m_{1}\right)\right)+m^{2}\left(\bar{\phi}_{\overline{3}}-m_{1}\right) \\
& +2 h \phi_{1}\left[h \bar{\phi}_{\overline{1}}\left(2 \bar{\phi}_{\overline{3}}-m_{1}\right)+m \bar{\phi}_{\overline{2}}\right] \\
0=\frac{\partial V}{\partial \bar{\phi}_{\overline{1}}}= & h\left(2 \bar{\phi}_{\overline{3}}-m_{1}\right)\left[h \phi_{1}\left(2 \phi_{3}-m_{1}\right)+m \phi_{2}\right] \\
& -\operatorname{det} C 2 h\left[h \bar{\phi}_{\overline{1}}\left(2 \bar{\phi}_{\overline{3}}-m_{1}\right)+m \bar{\phi}_{\overline{2}}\right] h\left(2 \bar{\phi}_{\overline{3}}-m_{1}\right)\left(h \bar{\phi}_{\overline{3}}\left(\bar{\phi}_{\overline{1}}-m_{1}\right)\right) \\
0=\frac{\partial V}{\partial \bar{\phi}_{\overline{2}}}= & m\left[h \phi_{1}\left(2 \phi_{3}-m_{1}\right)+m \phi_{2}\right] \\
& -\operatorname{det} C 2 h m\left[h \bar{\phi}_{\overline{1}}\left(2 \bar{\phi}_{\overline{3}}-m_{1}\right)+m \bar{\phi}_{\overline{2}}\right]\left(h \bar{\phi}_{\overline{3}}\left(\bar{\phi}_{\overline{3}}-m_{1}\right)\right) \\
0=\frac{\partial V}{\partial \bar{\phi}_{\overline{3}}}= & \left.2 h \bar{\phi}_{\overline{3}}-h m_{1}\right)\left(h \phi_{3}\left(\phi_{3}-m_{1}\right)\right)+(m)^{2}\left(\phi_{3}-m_{1}\right) \\
& +2 h \bar{\phi}_{1}\left[h \phi_{1}\left(2 \phi_{3}-m_{1}\right)+m \phi_{2}\right] \\
& -\operatorname{det} C h 2\left[h \bar{\phi}_{\overline{1}}\left(2 \bar{\phi}_{\overline{3}}-m_{1}\right)+m \bar{\phi}_{\overline{2}}\right] 2 h \bar{\phi}_{1} h \bar{\phi}_{\overline{3}}\left(\bar{\phi}_{\overline{3}}-m_{1}\right) \\
& -\operatorname{det} C h\left[h \bar{\phi}_{\overline{1}}\left(2 \bar{\phi}_{\overline{3}}-m_{1}\right)+m \bar{\phi}_{\overline{2}}\right]^{2}\left(2 h \bar{\phi}_{\overline{3}}-h m_{1}\right)
\end{aligned}
$$

As in the previous example, let us first consider the case $m \neq 0$. In that case, eq. $(3.20$ ) implies

$$
h \bar{\phi}_{\overline{1}}\left(2 \bar{\phi}_{\overline{3}}-m_{1}\right)+m \bar{\phi}_{\overline{2}}=0
$$

Again, the l.h.s of this equation appears as a factor in all terms containing $\operatorname{det} C$ and hence all dependence on $C_{\alpha \beta}$ disappears and field configurations corresponding to extrema of the potential are not affected by the deformation. Moreover, the value of the potential is also unaffected by non(anti)commutativity since terms containing $\operatorname{det} C$ are multiplied by the same vanishing factor. As explained in [10] there are supersymmetric vacua $\phi_{i}^{S}$ which corresponds to

$$
\phi_{3}^{S}=m_{1}, \quad \phi_{2}^{S}=-\frac{h m_{1}}{m} \phi_{1}^{S}
$$

(in the deformed case we should have identical values for fields $\bar{\phi}_{\bar{i}}$ which, in the deformed case are not automatically related to $\phi_{i}$ ).

As in the undeformed case, there are also extrema $\phi^{M}$ for which $V\left[\phi^{M}\right] \neq 0$. In fact, the Euclidean $V\left[\phi^{M}\right]$ is a real negative number which in Minkowski undeformed superspace would lead to the metastable vacua. The explicit form of the solutions is the same as in the undeformed case.

Let us now consider the $m=0$ case. In the undeformed (Minkowski) space, the nonsupersymmetric (metastable) vacua present for $m \neq 0$ are lost but, as we will see, the situation changes in the deformed case. Indeed for vanishing $m$ the scalar potential takes the form

$$
\begin{aligned}
V_{E}= & -h \phi_{3}\left(\phi_{3}-m_{1}\right) h \bar{\phi}_{\overline{3}}\left(\bar{\phi}_{\overline{3}}-m_{1}\right) \\
& -h \phi_{1}\left(2 \phi_{3}-m_{1}\right) h \bar{\phi}_{\overline{1}}\left(2 \bar{\phi}_{\overline{3}}-m_{1}\right) \\
& +\operatorname{det} C h^{2}\left[h \bar{\phi}_{\overline{1}}\left(2 \bar{\phi}_{\overline{3}}-m_{1}\right)\right]^{2} \bar{\phi}_{\overline{3}}\left(\bar{\phi}_{\overline{3}}-m_{1}\right)
\end{aligned}
$$


Let us compare the supersymmetric vacuum states between the undeformed and the deformed case. In the undeformed case, we have four supersymmetric vacuum states:

$$
\begin{array}{rlrl}
\phi_{1} & =\bar{\phi}_{\overline{1}}=\phi_{3}=\bar{\phi}_{\overline{3}}=0 & \\
\phi_{1}=\bar{\phi}_{\overline{1}}=\phi_{3}=0, & \bar{\phi}_{\overline{3}}=m_{1} \\
\phi_{1}=\bar{\phi}_{\overline{1}}=\bar{\phi}_{\overline{3}}=0, & \phi_{3}=m_{1} \\
\phi_{1}=\bar{\phi}_{\overline{1}}=0, & \phi_{3}=\bar{\phi}_{\overline{3}}=m_{1}
\end{array}
$$

In the deformed case, the vacua (3.28) are still present. In addition, there are other four supersymmetric vacua:

$$
\begin{array}{lll}
\phi_{1}=\bar{\phi}_{\overline{3}}=0, & \bar{\phi}_{\overline{1}}= \pm \frac{i}{2 h \sqrt{-\operatorname{det} C}}, & \phi_{3}=m_{1} / 2 \\
\phi_{1}=\bar{\phi}_{\overline{3}}=0, & \bar{\phi}_{\overline{1}}= \pm \frac{i}{2 h \sqrt{-\operatorname{det} C}}, & \phi_{3}=m_{1}
\end{array}
$$

As in the case of the extrema (3.16) of the previous example, in the limit $\operatorname{det} C \rightarrow 0^{+}$these extrema correspond to runaway directions which do not exist in the case of the undeformed potential $\operatorname{det} C=0$.

Concerning the supersymmetry breaking vacua, there is no difference between the undeformed and deformed case, having in both the pseudomoduli space:

$$
\phi_{3}=\bar{\phi}_{3}=m_{1} / 2
$$

for which $V=\left(m_{1} / 2\right)^{4} h^{2}$ in the undeformed case and $V_{E}=-\left(m_{1} / 2\right)^{4} h^{2}$ in the deformed one.

\subsection{A more general superpotential}

We end this section discussing conditions on a general cubic superpotential under which the vacuum structure remains unaffected by the deformation. Consider $n$ chiral superfields $\Phi_{i}(i=1,2, \ldots, n)$, a canonical Kähler potential and a deformed superpotential of the form

$$
\mathcal{W}\left(\Phi_{p}\right)=C+C_{q} \Phi_{q}+C_{q r} \Phi_{q} * \Phi_{r}+C_{q r s} \Phi_{q} * \Phi_{r} * \Phi_{s}
$$

with $C, C_{q}, C_{q r}$, y $C_{q r s}$ arbitrary coefficients, symmetric in all their indices. As before, in view of the form of the superpotential, the functional $\mathcal{W}\left(\phi_{i}+\xi c F_{i}\right)$, as defined in (2.16), will just contain terms with powers $0,1,2$, and 3 of $\xi$. Only even powers will contribute to $\tilde{\mathcal{W}}$ obtaining

$$
\begin{aligned}
\mathcal{W}\left(\phi_{i}+\xi c F_{i}\right)= & C+C_{q} \phi_{q}+C_{q r} \phi_{q} \phi_{r}+C_{q r s} \phi_{q} \phi_{r} \phi_{s} \\
& +\xi^{2} c^{2}\left(C_{q r} F_{q} F_{r}+3 C_{q r s}\left(\phi_{q} F_{r} F_{s}\right)\right) \\
\widetilde{\mathcal{W}}\left(\phi_{i}, F_{i}\right)= & 2\left(C+C_{q} \phi_{q}+C_{q r} \phi_{q} \phi_{r}+C_{q r s} \phi_{q} \phi_{r} \phi_{s}\right) \\
& +\frac{2 c^{2}}{3}\left(C_{q r} F_{q} F_{r}+3 C_{q r s}\left(\phi_{q} F_{r} F_{s}\right)\right)
\end{aligned}
$$


Using the equations of motion for auxiliary fields $\bar{F}_{\bar{j}}$ we find $F_{i}=-\partial \overline{\mathcal{W}} / \partial \bar{\phi}_{i}$ and then

$$
\widetilde{\mathcal{W}}_{i}=2\left(C_{i}+2 C_{i r} \phi_{r}+3 C_{i r s} \phi_{r} \phi_{s}\right)+2 c^{2} C_{i r s} F_{r} F_{s}
$$

With this

$$
V=\left.F_{i}\left[C_{i}+2 C_{i r} \phi_{r}+3 C_{i r s} \phi_{r} \phi_{s}+c^{2} C_{i r s} F_{r} F_{s}\right]\right|_{F_{i}=F_{i}\left(\phi_{j}, \bar{\phi}_{\bar{j}}\right)}
$$

The extrema conditions are

$$
\begin{aligned}
& 0=\frac{\partial V}{\partial \phi_{j}}=\left.2 F_{i}\left[C_{i j}+3 C_{i j r} \phi_{r}\right]\right|_{F_{i}=F_{i}\left(\phi_{j}, \bar{\phi}_{\bar{j}}\right)} \\
& 0=\frac{\partial V}{\partial \bar{\phi}_{\bar{j}}}=F_{i},\left.\bar{j}\left[C_{i}+2 C_{i r} \phi_{r}+3 C_{i r s} \phi_{r} \phi_{s}+3 c^{2} C_{i r s} F_{r} F_{s}\right]\right|_{F_{i}=F_{i}\left(\phi_{j}, \bar{\phi}_{\bar{j}}\right)}
\end{aligned}
$$

Suppose that the following relations among coefficients $C_{i j}$ and $C_{i j r}$ hold

$$
\left(C_{i j}+3 C_{i j r} \phi_{r}\right)=\delta_{i a} M_{j}+\delta_{j a} M_{i}
$$

for some value $a$ ( $M_{i}$ is an arbitrary, field dependent, vector). Such conditions imply that $F_{a}=0$ (unless, for all $i$, the pairs of coefficients $\left(C_{i}, C_{i a a}\right)$ are proportional to each other, cf. (3.36) ). If we still impose a more restrictive condition on $C_{i j r}$, namely that it vanishes unless it has two indices $a$, we see that the extrema conditions (3.37) are independent of $\operatorname{det} C$ and also the potential at the extrema is unaffected by the deformation.

Is easy to see that the above mentioned conditions force the potential to take the form

$$
\mathcal{W}=\sum_{i \neq a} \Phi_{i} * g_{i}\left(\Phi_{a}\right)+\mathrm{ST}
$$

with $g_{i}$ quadratic functions, not all proportional to each other.

By the above arguments, the vacuum structure of this superpotential is not deformed. Note that the explicit examples previously discussed in subsection 3.2 belong (for $m \neq 0$ ) to this class of potentials, insensitive to the deformations.

\section{Noncanonical deformed Kähler potentials}

As a first simple example of noncanonical Kähler potential we consider

$$
K=(\Phi * \bar{\Phi})^{2}
$$

In this case eqs. (2.18) and (2.19) take the form

$$
\begin{aligned}
Z(\phi, \bar{\phi}, F)= & \int_{-1}^{1} d \xi(\phi \bar{\phi}+\xi c F \bar{\phi})^{2}=2(\phi \bar{\phi})^{2}+\frac{2}{3} c^{2}(\bar{\phi} F)^{2} \\
Y(\phi, \bar{\phi}, F, \bar{F})= & \bar{F}\left(4 \phi^{2} \bar{\phi}+\frac{4}{3} c^{2} F^{2} \bar{\phi}\right)-\frac{1}{2}(\bar{\chi} \bar{\chi})\left(4 \phi^{2}+\frac{4}{3} c^{2} F^{2}\right) \\
& +c \int_{-1}^{1} d \xi \xi\left[\partial^{\mu} \bar{\phi} \partial_{\mu} \bar{\phi}\left(2 \phi^{2}+4 \xi c \phi F+2 \xi^{2} c^{2} F^{2}\right)\right. \\
& +2 \square \bar{\phi}(\phi \bar{\phi}+\xi c F \bar{\phi})(\phi+\xi c F)]
\end{aligned}
$$




$$
\begin{aligned}
= & \bar{F}\left(4 \phi^{2} \bar{\phi}+\frac{4}{3} c^{2} F^{2} \bar{\phi}\right)-\frac{1}{2}(\bar{\chi} \bar{\chi})\left(4 \phi^{2}+\frac{4}{3} c^{2} F^{2} \bar{\phi}\right) \\
& +\left(\frac{4}{3} c^{2} \phi F\right)\left[2 \partial^{\mu} \bar{\phi} \partial_{\mu} \bar{\phi}+\bar{\phi} \square \bar{\phi}\right]
\end{aligned}
$$

so that the kinetic part of the component field Lagrangian reads

$$
\begin{aligned}
L_{K}= & -4 \bar{F} F \phi \bar{\phi}+2 F(\bar{\chi} \bar{\chi}) \phi+\frac{2}{3} \operatorname{det} C F^{2}\left[2 \partial^{\mu} \bar{\phi} \partial_{\mu} \bar{\phi}+\bar{\phi} \square \bar{\phi}\right] \\
& -\frac{1}{2} \partial^{\mu} \bar{\phi} \partial_{\mu} \bar{\phi}\left(4 \phi^{2}-\frac{4}{3} \operatorname{det} C F^{2}\right)-\frac{1}{2} \square \bar{\phi}\left(4 \phi^{2} \bar{\phi}+\frac{4}{3} c^{2} F^{2} \bar{\phi}\right) \\
& -\frac{1}{4} \chi \chi(8 \bar{F} \bar{\phi}-4 \bar{\chi} \bar{\chi})+\frac{1}{2} i\left(\chi \sigma^{\mu} \bar{\chi}\right) \partial_{\mu} \bar{\phi} 8 \phi+\frac{1}{2} i\left(\chi \sigma^{\mu} \partial_{\mu} \bar{\chi}\right) 8 \phi \bar{\phi}
\end{aligned}
$$

Since $\operatorname{det} C$ only affects kinetic energy terms for $\bar{\phi}$, the scalar potential for this noncanonical Kähler potential could only be deformed by contributions arising from the superpotential.

Because $L_{K}$ is a linear functional of the Kähler potential, the discussion above

$$
K=\Phi * \bar{\Phi}+\lambda(\Phi * \bar{\Phi})^{2}
$$

Such a Kähler potential can be though as resulting from the approximation of a general potential $K_{*}(\Phi, \bar{\Phi})=f(\Phi * \bar{\Phi})$ for $\Phi \approx 0$. Then, in the weak-field regime we have to expect that only the deformation of the superpotential would affect the vacuum structure.

Modifications arise for Kähler potentials with higher powers, namely $(\bar{\Phi} \Phi)^{n}$ with $n>2$. Consider the simplest case $n=3$,

$$
K_{3}=(\bar{\Phi} * \Phi)^{3}
$$

Since we are interested in purely bosonic contributions with no derivatives, we will restrict our analysis to these type of terms which will be indicated with the subscript "boson". We have,

$$
\begin{aligned}
Z(\phi, \bar{\phi}, F) & =\int_{-1}^{1} d \xi(\phi \bar{\phi}+\xi c F \bar{\phi})^{3}=2\left[(\phi \bar{\phi})^{3}-\operatorname{det} C \bar{\phi}^{3} \phi F^{2}\right] \\
Y_{\text {boson }}(\phi, \bar{\phi}, F, \bar{F}) & =6 \bar{F}\left[\phi^{3} \bar{\phi}^{2}-\operatorname{det} C \bar{\phi}^{2} \phi F^{2}\right] \\
\frac{\partial Y_{\text {boson }}}{\partial \phi} & =6 \bar{F}\left[3 \phi^{2} \bar{\phi}^{2}-\operatorname{det} C \bar{\phi}^{2} F^{2}\right]
\end{aligned}
$$

The corresponding contribution to the Lagrangian is,

$$
\left.L_{K_{3}}\right|_{\text {boson }}=3 F \bar{F} \bar{\phi}^{2}\left(3 \phi^{2}-\operatorname{det} C F^{2}\right)
$$

so the relevant parts of the equations of motion for the auxiliary fields are

$$
\begin{array}{r}
3 F \bar{\phi}^{2}\left(3 \phi^{2}-\operatorname{det} C F^{2}\right)+\frac{\partial \overline{\mathcal{W}}}{\partial \bar{\phi}}=0 \\
9 \bar{F} \bar{\phi}^{2} \phi^{2}-9 \operatorname{det} C F^{2} \bar{F} \bar{\phi}^{2}+\frac{\partial \mathcal{W}}{\partial \phi}=0
\end{array}
$$


We then conclude that both $F$ and $\bar{F}$ will depend on $\operatorname{det} C$ independently of the choice of the superpotential, so that for a Kähler potential cubic in $\bar{\Phi} * \Phi$ the scalar potential and, a fortiori, the vacuum structure will be affected by the deformation.

Let us consider a simple example that illustrates the discussion above. It corresponds to superpotentials $\mathcal{W}$ and $\overline{\mathcal{W}}$ (recall that in Euclidean space, they are independent functionals)

$$
\mathcal{W}=\frac{1}{2} f \Phi * \Phi \quad, \quad \overline{\mathcal{W}}=g
$$

and the Kähler potential defined in (4.4).

Given superpotentials (4.8) we get for the auxiliary fields, using eqs. of motion (4.6) and (4.7),

$$
\begin{aligned}
& F=\frac{i \sqrt{3} \phi}{\operatorname{det} C} \\
& \bar{F}=\frac{f}{36 \phi \bar{\phi}^{2}}
\end{aligned}
$$

It can be seen from eq. (2.17) that, as expected, the scalar potential is affected by the deformation of the Kähler potential through the dependence of $F$ on $\operatorname{det} C$ as given by (4.9).

Let us end this section by pointing that a completely analogous behavior can be found for a Kähler potential of the form $K_{n}=(\bar{\Phi} * \Phi)^{n}$. For example, for odd $n$ we find, instead of eq. (4.6), that the auxiliary field $F$ obeys the equation

$$
\frac{n}{2 \sqrt{-\operatorname{det} C}} \bar{\phi}^{n-1}\left((\phi+\sqrt{-\operatorname{det} C} F)^{n}-(\phi-\sqrt{-\operatorname{det} C} F)^{n}\right)+\frac{\partial \bar{W}}{\partial \bar{\phi}}=0
$$

This is a degree $n$ polynomial equation for $F$, with coefficients $\operatorname{depending}$ on $\operatorname{det} C$ as a result of the deformation in the Kähler potential. The solution for $F$ will be in general $\operatorname{det} C$-dependent (as we have explicitly seen for the particular case $n=3$ ) and hence the scalar potential as given by (2.17) will in turn be deformed.

\section{Discussion}

In this work we have discussed the vacuum structure of $\mathcal{N}=1 / 2$ supersymmetric theories of chiral superfields in deformed superspace. We have analyzed O'Raifeartaigh models with general deformed superpotentials, including the case in which the Kähler potential is non-canonical. The question we intended to clarify was how the landscape of extrema of the classical scalar potential is affected by a deformation of superspace.

As explained in section 2.3, although hermiticity of the theory is lost because of the deformation, the analysis of the critical points of the resulting complex potential allows to decide whether the $\mathcal{N}=1 / 2$ supersymmetry surviving the deformation is spontaneously unbroken. In fact, as we have seen, loss of hermiticity implies that the scalar potential is in principle complex and, moreover, because superfields $\bar{\Phi}_{i}$ are not the complex conjugate of $\Phi_{i}$, scalars $\bar{\phi}_{\bar{i}}$ do not in general coincide with $\phi_{i}^{*}$. This of course complicates the analysis of extrema of the potential unless we impose some restrictions on fields and potentials. 
Restricting the analysis to the case of field configurations such that $\bar{\phi}_{\bar{i}}=\phi_{i}^{*}$, we have seen in section 3.2 that the vacuum configurations for superpotentials (3.1) and (3.17) described in [9] 10] for undeformed superspace, are also present in the deformed case when the coefficient $m \neq 0$. Hence in both cases there is symmetry breaking and a classical pseudomoduli space with degenerate non supersymmetric vacua. The difference between the two cases is that in the latter there can be metastable (for an appropriate choice of coefficients) vacua which are absent in the former.

An interesting phenomenon takes place for $m=0$ : in the limit $\operatorname{det} C \rightarrow 0$, in which the deformation vanishes, there are additional extrema, eqs. (3.16) and (3.29), that correspond to runaway directions which do not exist in the case of the undeformed potential $\operatorname{det} C=0$. This phenomenon is resemblant of what happens with solitons in $\theta$-deformed noncommutative space: apart from those that reproduce the ordinary regular solitons in the $\theta \rightarrow 0$ limit, there are "fluxon" solutions with no regular counterpart in ordinary space (see 19] and reference therein).

In section 3.3 we considered a general cubic superpotential (which encompasses the two previous examples) and found the conditions under which the vacuum structure remains unaffected by the deformation.

We also considered non-canonical deformed Kähler potentials which, being nonquadratic, could be expected to induce a $C$-dependence on the vacuum structure. The case $K=(\bar{\Phi} * \Phi)^{2}$ is a counterexample of this possibility since we proved that only the kinetic energy is affected by the deformation. Hence, in a weak-field approximation, the vacuum dependence on the $C$-deformation will only enter through the deformed superpotential. We need higher powers $(n>2)$ of $\bar{\Phi} * \Phi$ in order to change the vacuum structure as we have explicitly shown at the end of section 4 .

The discussion in this work is valid at tree-level, and should be corrected by including leading quantum corrections to the potential. Being the theory non-hermitian, one should resort to complex saddle point or steepest descent methods. We hope to report on this issue in a following investigation. O'Raifeartaigh-type models, as those considered here, can arise naturally and dynamically in the low-energy limit of simple SUSY gauge theories. In this respect, the extension of the analysis we have presented to the case of deformed super Yang-Mills theory is also a subject we hope to address in the future.

\section{Acknowledgments}

This work was partially supported by PIP6160-CONICET, BID 1728OC/AR PICT20204ANPCYT grants and by CIC and UNLP, Argentina.

\section{References}

[1] J. de Boer, P.A. Grassi and P. van Nieuwenhuizen, Non-commutative superspace from string theory, Phys. Lett. B 574 (2003) 98 hep-th/0302078.

[2] H. Ooguri and C. Vafa, Gravity induced C-deformation, Adv. Theor. Math. Phys. 7 (2004) 405 hep-th/0303063. 
[3] N. Seiberg, Noncommutative superspace, $N=1 / 2$ supersymmetry, field theory and string theory, JHEP 06 (2003) 010 hep-th/0305248.

[4] T. Araki, K. Ito and A. Ohtsuka, Supersymmetric gauge theories on noncommutative superspace, Phys. Lett. B 573 (2003) 209 hep-th/0307076.

[5] O. Lunin and S.-J. Rey, Renormalizability of non(anti)commutative gauge theories with $N=1 / 2$ supersymmetry, JHEP 09 (2003) 045 hep-th/0307275.

[6] R. Britto, B. Feng and S.-J. Rey, Deformed superspace, $N=1 / 2$ supersymmetry and (non) renormalization theorems, JHEP 07 (2003) 067 hep-th/0306215;

Non(anti)commutative superspace, UV/IR mixing and open Wilson lines, JHEP 08 (2003) 001 hep-th/0307091.

[7] R. Britto and B. Feng, $N=1 / 2$ Wess-Zumino model is renormalizable, Phys. Rev. Lett. 91 (2003) 201601 hep-th/0307165.

[8] L. O'Raifeartaigh, Spontaneous symmetry breaking for chiral scalar superfields, Nucl. Phys. B 96 (1975) 331.

[9] K. Intriligator, N. Seiberg and D. Shih, Dynamical SUSY breaking in meta-stable vacua, JHEP 04 (2006) 021 hep-th/0602239.

[10] K. Intriligator and N. Seiberg, Lectures on supersymmetry breaking, Class. and Quant. Grav. $24(2007)$ S741.

[11] D. Shih, Spontaneous R-symmetry breaking in O'Raifeartaigh models, JHEP 02 (2008) 091 hep-th/0703196.

[12] S.P. de Alwis, On gauge mediated SUSY breaking and moduli stabilization, Phys. Rev. D 76 (2007) 086001.

[13] K. Intriligator, N. Seiberg and D. Shih, Supersymmetry breaking, R-symmetry breaking and metastable vacua, JHEP 07 (2007) 017 hep-th/0703281.

[14] S. Ray, Some properties of meta-stable supersymmetry-breaking vacua in Wess-Zumino models, Phys. Lett. B 642 (2006) 137 hep-th/0607172; Supersymmetric and $R$ symmetric vacua in Wess-Zumino models, arXiv:0708.2200.

[15] T. Hatanaka, S.V. Ketov, Y. Kobayashi and S. Sasaki, $N=1 / 2$ supersymmetric four-dimensional non-linear $\sigma$-models from non-anti-commutative superspace, Nucl. Phys. B 726 (2005) 481 hep-th/0506071.

[16] L. Álvarez-Gaumé and M.A. Vazquez-Mozo, On nonanticommutative $N=2 \sigma$-models in two dimensions, JHEP 04 (2005) 007 hep-th/0503016.

[17] T. Hatanaka, S. Ketov and S. Sasaki, Summing up NAC-deformed Kähler potential, Phys. Lett. B 619 (2005) 352 hep-th/0504191.

[18] C.-S. Chu and T. Inami, Konishi anomaly and central extension in $N=1 / 2$ supersymmetry, Nucl. Phys. B 725 (2005) 327 hep-th/0505141.

[19] F.A. Schaposnik, Noncommutative solitons and instantons, Braz. J. Phys. 34 (2004) 1349 hep-th/0310202, also in Minneapolis 2004, continuous advances in QCD, T. Gherghetta ed., World Scientific, Singapore (2004). 